\title{
Dipolar energy of Nd-Fe-B nanocrystalline magnets in magnetization reversal process
}

\author{
Hiroyuki Ohtori, ${ }^{1, a)}$ Kaoru Iwano, ${ }^{1}$ Chiharu Mitsumata, ${ }^{2}$ Masao Yano, ${ }^{3}$ Akira Kato, ${ }^{3}$ \\ Tetsuya Shoji, ${ }^{3}$ Akira Manabe, ${ }^{3}$ and Kanta Ono ${ }^{1}$ \\ ${ }^{1}$ Institute of Materials Structure Science, High Energy Accelerator Research Organization (KEK), \\ Tsukuba, Ibaraki 305-0801, Japan \\ ${ }^{2}$ National Institute of Materials Science, Tsukuba, Ibaraki 305-0047, Japan \\ ${ }^{3}$ TOYOTA Motor Corporation, Toyota, Aichi 471-8571, Japan
}

(Presented 5 November 2013; received 22 September 2013; accepted 4 November 2013; published online 4 February 2014)

\begin{abstract}
We analyzed the dipolar energy of $\mathrm{Nd}-\mathrm{Fe}-\mathrm{B}$ nanocrystalline magnets in magnetization reversal process through visualizing magnetic dipolar interaction. We obtained magnetization distribution images experimentally by using scanning transmission X-ray microscopy (STXM). The magnetic dipolar interaction was calculated by the interaction between the magnetization at each point and those at the other points on the STXM image. We showed the dipolar energy in the nanocrystalline $\mathrm{Nd}-\mathrm{Fe}-\mathrm{B}$ magnets and compared it with the exchange energy at various applied fields. Our results indicated the significance of the dipolar energy in magnetization reversal process. (C) 2014 AIP Publishing LLC. [http://dx.doi.org/10.1063/1.4864041]
\end{abstract}

Recently, Nd-Fe-B magnets have been attracting much attention with the increase in the consumption of them ${ }^{1}$ and have been intensively examined using Kerr microscopy, ${ }^{2-10}$ magnetic force microscopy, ${ }^{3,11-17}$ Bitter method, ${ }^{17-19}$ scanning electron microscopy (SEM), ${ }^{20,21}$ Lorenz microscopy, ${ }^{13,22-25}$ and electron holography. ${ }^{24,26}$ In nanocrystalline Nd-Fe-B magnets, whose particle size is less than the single-domain size, magnetic dipolar interactions become essential in magnetization reversal processes. However, the significance of dipolar energy in these magnets is not yet understood, because a quantitative measurement of the dipolar energy in magnetic materials is difficult. Therefore, we consider that it is important to analyze the dipolar energy for revealing the nature of the magnetization reversal processes. In order to analyze the dipolar energy, we demonstrate a visualization of the magnetic dipolar interaction in permanent magnets using scanning transmission X-ray microscopy (STXM). Essentially, STXM is a microscope that takes advantage of the X-ray magnetic circular dichroism (XMCD) effect. Since the XMCD effect is proportional to the magnetization of a specific element and is sensitive to the projection of the magnetization along the direction of photon propagation, the STXM image of the magnetic contrast shows the real space distribution of element-specific magnetization with high spatial resolution. Therefore, STXM is a powerful tool to investigate the magnetic microstructure of $\mathrm{Nd}-\mathrm{Fe}-\mathrm{B}$ magnet. $^{27}$ In this paper, we analyzed the STXM image of the magnetization distribution with a special attention to the direction perpendicular to the surface. We calculated the potential energy of the magnetic dipolar interaction between the magnetization at each point and those at the other points on the STXM image in order to obtain the distribution of the dipolar energy. Finally, we visualized the magnetic dipolar interaction in the nanocrystalline $\mathrm{Nd}-\mathrm{Fe}-\mathrm{B}$ magnets and

\footnotetext{
a) Author to whom correspondence should be addressed. Electronic mail: hiroyuki.otori@kek.jp.
}

compared it with the exchange energy at remanent states after applying $0,1,2,4,6$, and $10 \mathrm{kOe}$.

In order to visualize the magnetic dipolar interaction energies, we have to calculate it from the magnetization distribution which can be obtained from STXM image. ${ }^{28}$ Potential energy of the magnetic dipolar interaction $U$ is given by

$$
U=-\int \frac{\nabla \cdot \boldsymbol{M}\left(\boldsymbol{r}^{\prime}\right)}{\left|\boldsymbol{r}-\boldsymbol{r}^{\prime}\right|} d V^{\prime}+\int \frac{\boldsymbol{n} \cdot \boldsymbol{M}\left(\boldsymbol{r}^{\prime}\right)}{\left|\boldsymbol{r}-\boldsymbol{r}^{\prime}\right|} d S^{\prime}
$$

In this study, we assume the depth of sample is thin enough to calculate the magnetization as $\boldsymbol{M}=(0,0, m(x, y))$. By substituting $\boldsymbol{M}=(0,0, m(x, y))$ into Eq. (1), we obtain

$$
\begin{aligned}
U= & -\int d V^{\prime} \frac{\partial}{\partial z^{\prime}} \frac{m\left(x^{\prime}, y^{\prime}\right)}{\left|\boldsymbol{r}-\boldsymbol{r}^{\prime}\right|} \\
& +\int d S^{\prime} \int d z^{\prime} \frac{\partial}{\partial z^{\prime}} \frac{m\left(x^{\prime}, y^{\prime}\right)}{\sqrt{\left(x-x^{\prime}\right)^{2}+\left(y-y^{\prime}\right)^{2}+\left(z-z^{\prime}\right)^{2}}} \\
= & \int_{-a}^{a} \int_{-b}^{b} \int_{-c}^{c} \frac{\left(z-z^{\prime}\right) m\left(x^{\prime}, y^{\prime}\right) d x^{\prime} d y^{\prime} d z^{\prime}}{\left(\left(x-x^{\prime}\right)^{2}+\left(y-y^{\prime}\right)^{2}+\left(z-z^{\prime}\right)^{2}\right)^{\frac{3}{2}}},
\end{aligned}
$$

where $\boldsymbol{r}=(x, y, z)$ is the coordinate of the observation point, $\boldsymbol{r}^{\prime}=\left(x^{\prime}, y^{\prime}, z^{\prime}\right)$ is the coordinate of the source point, $2 a$ is the width of a sample in $x$ direction, $2 b$ is the width of the sample in $y$ direction, and $2 c$ is the thickness of the sample. The relation between the magnetic dipolar interaction energy $E_{d}$ and the magnetic field $\boldsymbol{H}$ is given by

$$
E_{d}=-\frac{1}{2} \int \boldsymbol{M} \cdot \boldsymbol{H} d V=-\frac{1}{2} \int m(x, y) H_{z} d V,
$$

where $H_{z}$ is the $z$ component of $\boldsymbol{H}$. On the other hand, the relation between $U$ and $\boldsymbol{H}$ is given by $\boldsymbol{H}=-\nabla U$. Therefore, we can obtain the $E_{d}$ as the function of $U$ 
(a)

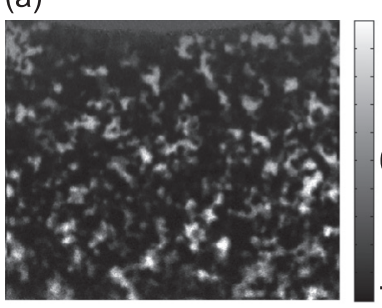

$$
\begin{aligned}
E_{d} & =\frac{1}{2} \int d x \int \operatorname{dym}(x, y) \int d z \frac{\partial}{\partial z} U(x, y, z) \\
& =\frac{1}{2} \int d x \int \operatorname{dym}(x, y)(U(x, y, c)-U(x, y,-c)) .
\end{aligned}
$$

From Eq. (2), the term of $U$ in Eq. (4) is given by

$$
\begin{aligned}
& U(x, y, c)-U(x, y,-c) \\
& =\int d x^{\prime} \int d y^{\prime} m\left(x^{\prime}, y^{\prime}\right) \\
& \quad \times \int d z^{\prime}\left[\frac{c-z^{\prime}}{\left(r_{x y}^{2}+\left(c-z^{\prime}\right)^{2}\right)^{\frac{3}{2}}}-\frac{-c-z^{\prime}}{\left(r_{x y}^{2}+\left(-c-z^{\prime}\right)^{2}\right)^{\frac{3}{2}}}\right] \\
& =\int d x^{\prime} \int d y^{\prime} m\left(x^{\prime}, y^{\prime}\right) 2\left[\frac{1}{r_{x y}}-\frac{1}{\sqrt{r_{x y}^{2}+(2 c)^{2}}}\right],
\end{aligned}
$$

where $r_{x y}=\sqrt{\left(x-x^{\prime}\right)^{2}+\left(y-y^{\prime}\right)^{2}}$ is the length between the observation point and the source point. By using Eqs. (4) and (5), we can visualize the distribution of the dipolar energy as shown in Fig. 1(b) from the STXM image of the magnetization distribution as shown in Fig. 1(a).

By using the method shown in Fig. 1, we visualize the magnetic dipolar interaction in the nanocrystalline $\mathrm{Nd}-\mathrm{Fe}-\mathrm{B}$ magnet from the STXM image. We can obtain the twodimensional magnetic dipolar interaction energy $E_{d_{x y}}$, which also represents the density of the magnetic dipolar interaction energy $E_{d}$ at $(x, y)$, by substituting Eq. (5) into Eq. (4)

$$
E_{d_{x y}}(x, y)=\frac{1}{2} \frac{m(x, y)}{2 c} \iint d x^{\prime} d y^{\prime} g\left(\boldsymbol{x}, \boldsymbol{x}^{\prime}\right) m\left(x^{\prime}, y^{\prime}\right),
$$

where

$$
g\left(\boldsymbol{x}, \boldsymbol{x}^{\prime}\right) \equiv 2\left(\frac{1}{r_{x y}}-\frac{1}{\sqrt{r_{x y}^{2}+(2 c)^{2}}}\right)
$$

$\boldsymbol{x}=(x, y)$ is the coordinate of the observation point on the $x$ $y$ plane, and $\boldsymbol{x}^{\prime}=\left(x^{\prime}, y^{\prime}\right)$ is the coordinate of the source point on the same plane. In Fig. 2, we show the visualized magnetic dipolar interaction in nanocrystalline $\mathrm{Nd}-\mathrm{Fe}-\mathrm{B}$ magnet at remanent states after applying $0,1,2,4,6$, and $10 \mathrm{kOe}$. When the applied field approaches the coercivity field ( $\sim 10 \mathrm{kOe})$, the total dipolar energy decreases. This behavior suggests that the decreased dipolar energy contributes to the stability of permanent magnets against the applied field.

On the other hand, the excess exchange energy including only nearest neighbour interaction is given by
FIG. 1. (a) The STXM image of the magnetization distribution in nanocrystalline Nd-Fe-B magnet. The remanent magnetic flux density is $1.6 \mathrm{~T}$. (b) The distribution of the magnetic dipolar interaction energy obtained from (a). The thickness of the sample is $60 \mathrm{~nm}$.

$$
E_{\mathrm{ex}}=-2 S^{2} J_{0} \frac{1}{\Omega} \sum_{j \neq i} \cos \varphi_{i j}
$$

where $\Omega$ is the atomic volume per magnetic ion and $\varphi_{i j}$ is the angle between neighbouring magnetic moments. By using the magnetic moments $\boldsymbol{M}$ and the saturation magnetization $M_{s}, \cos \varphi_{i j}$ is represented by

$$
\cos \varphi_{i j}=\frac{\boldsymbol{M}_{i}}{M_{s}} \cdot \frac{\boldsymbol{M}_{j}}{M_{s}} .
$$

Because $\boldsymbol{M}_{j}$ can be developed into a Taylor series, $\boldsymbol{M}_{j} / M_{s}=\boldsymbol{M}_{i} / M_{s}+\left(\boldsymbol{r}_{i j} \cdot \nabla\right) \boldsymbol{M}_{i} / M_{s}+\frac{1}{2}\left(\boldsymbol{r}_{i j} \cdot \nabla\right) \boldsymbol{M}_{i} / M_{s}, \quad$ the excess exchange energy due to inhomogeneities of spin distributions ${ }^{29}$ is finally given by

$$
E_{\mathrm{ex}}=A \frac{|\nabla \boldsymbol{M}|^{2}}{M_{s}^{2}}
$$

where $\boldsymbol{r}_{i j}$ is the nearest neighbour distance, $\mathrm{A}$ is the exchange stiffness constant, and the linear terms and the nondiagonal terms of the quadratic terms disappear in summation. We

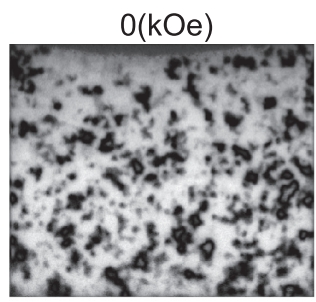

$2(\mathrm{kOe})$

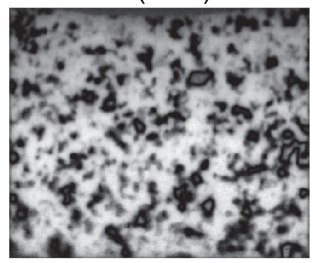

$6(\mathrm{kOe})$

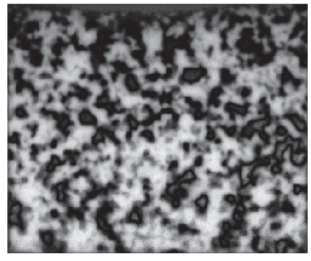

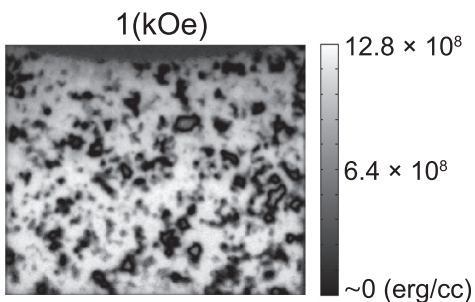

$4(\mathrm{kOe})$

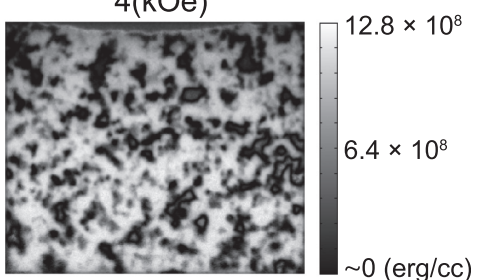

10(kOe)

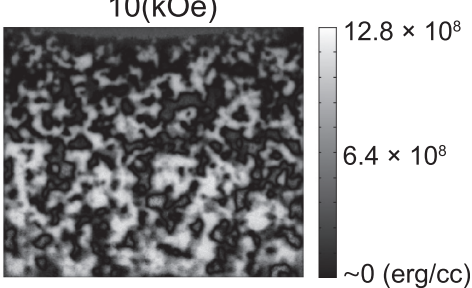

FIG. 2. The distribution of the dipolar energy for applied fields of $0,1,2,4$, 6 , and $10 \mathrm{kOe}$ in the nanocrystalline Nd-Fe-B magnet. The thickness of the sample is $60 \mathrm{~nm}$. The dipolar energy is high in white areas and low in black areas. 


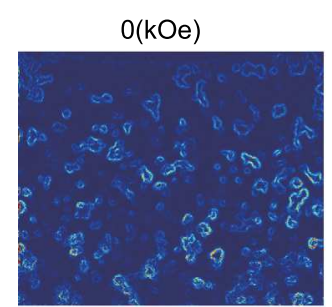

$2(\mathrm{kOe})$

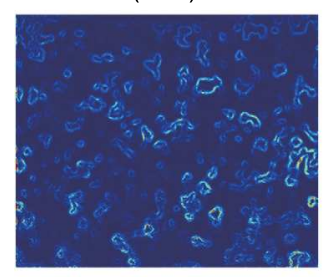

$6(\mathrm{kOe})$

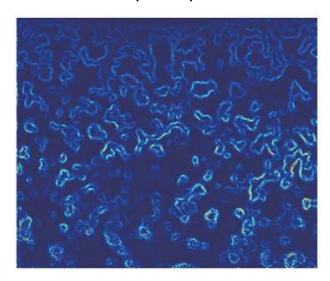

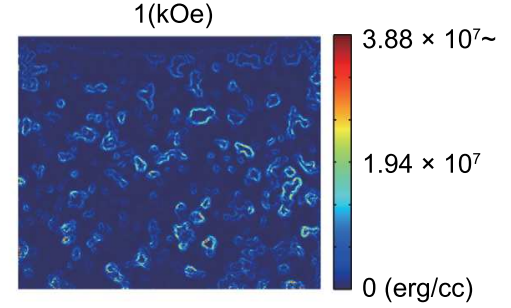

$4(\mathrm{kOe})$

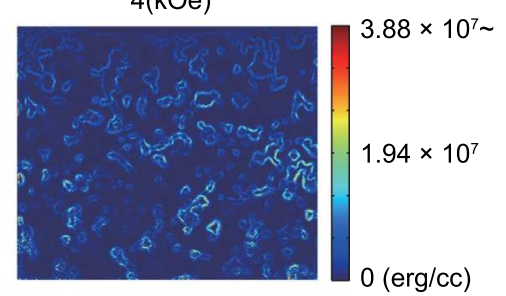

$10(\mathrm{kOe})$

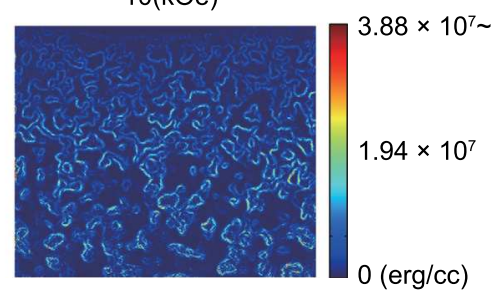

FIG. 3. The distribution of the excess exchange energy for applied fields of $0,1,2,4,6$, and $10 \mathrm{kOe}$ in the nanocrystalline Nd-Fe-B magnet. The exchange stiffness constant is $10 \mathrm{pJ} / \mathrm{m}$.

can obtain the excess exchange energy by substituting the value of the magnetization given by Fig. 1(a) to Eq. (10). Fig. 3 shows the distribution of the excess exchange energy in nanocrystalline $\mathrm{Nd}-\mathrm{Fe}-\mathrm{B}$ magnet at remanent states after applying 0, 1, 2, 4, 6, and $10 \mathrm{kOe}$. By comparing Fig. 3 with Fig. 2, we can confirm that, in magnetization reversal process, the amount of the exchange energy change is less than that of the dipolar energy change. Our results show that the magnetic dipolar interaction affected much more to the stability of permanent magnets than the exchange interaction because the radius of nanocrystalline magnets is less than single-domain radius therefore domain wall can not be created.

In summary, we visualized the magnetic dipolar interaction in a nanocrystalline Nd-Fe-B magnet using STXM. Using those images, we calculated the potential energy of the magnetic dipolar interaction between the magnetization at each point and those at the other points on the STXM image and obtained the distribution of the dipolar energy. We found that the total dipolar energy decreases when the applied field approaches the coercivity field ('10 kOe). We also found that, in magnetization reversal process, the amount of the exchange energy change is less than that of the dipolar energy change. Our results suggest that the magnetic dipolar interaction affected much more to the stability of permanent magnets against the applied field than the exchange interaction.

This research was supported by the Japan Science and Technology Agency (JST) under Collaborative Research Based on Industrial Demand "High Performance Magnets: Towards Innovative Development of Next Generation Magnets." Sample fabrication was partly performed under the Magnetic Materials for High-Efficiency Motors (MagHEM) project.

${ }^{1}$ O. Gutfleisch, M. A. Willard, E. Bruck, C. H. Chen, S. G. Sankar, and J. P. Liu, Adv. Mater. 23, 821 (2011).

${ }^{2}$ J. D. Livingston, J. Appl. Phys. 57, 4137 (1985).

${ }^{3}$ E. Zueco, W. Rave, R. Schafer, A. Hubert, and L. Schultz, J. Magn. Magn. Mater. 190, 42 (1998).

${ }^{4}$ R. Szymczak, D. Givord, and H. S. Li, Acta Phys. Pol., A 72, 113 (1987).

${ }^{5}$ L. Folks, R. Street, and R. C. Woodward, Appl. Phys. Lett. 65, 910 (1994).

${ }^{6}$ Y. G. Pastushenkov, A. Forkl, and H. Kronmuller, J. Magn. Magn. Mater. 174, 278 (1997).

${ }^{7}$ O. Gutfleisch, D. Eckert, R. Schafer, K. H. Muller, and V. Panchanathan, J. Appl. Phys. 87, 6119 (2000).

${ }^{8}$ K. Khlopkov, O. Gutfleisch, D. Eckert, D. Hinz, B. Wall, W. Rodewald, K. H. Muller, and L. Schultz, J. Alloys Compd. 365, 259 (2004).

${ }^{9}$ A. Kreyssig, R. Prozorov, C. D. Dewhurst, P. C. Canfield, R. W. McCallum, and A. I. Goldman, Phys. Rev. Lett. 102, 047204 (2009).

${ }^{10}$ M. Takezawa, K. Maruko, N. Tani, Y. Morimoto, J. Yamasaki, T. Nishiuchi, and S. Hirosawa, J. Appl. Phys. 107, 09A724 (2010).

${ }^{11}$ P. Grutter, E. Meyer, H. Heinzelmann, L. Rosenthaler, H. R. Hidber, and H. J. Guntherodt, J. Vac. Sci. Technol., A 6, 279 (1988).

${ }^{12}$ L. Folks, R. Street, R. C. Woodward, and K. Babcock, J. Magn. Magn. Mater. 159, 109 (1996).

${ }^{13}$ H. Lemke, T. Goddenhenrich, C. Heiden, and G. Thomas, IEEE Trans. Magn. 33, 3865 (1997).

${ }^{14}$ M. A. Al-Khafaji, W. M. Rainforth, M. R. J. Gibbs, H. A. Davies, and J. E. L. Bishop, J. Magn. Magn. Mater. 182, 111 (1998).

${ }^{15}$ V. Neu, S. Melcher, U. Hannemann, S. Fahler, and L. Schultz, Phys. Rev. B 70, 144418 (2004).

${ }^{16}$ T. G. Woodcock, K. Khlopkov, A. Walther, N. M. Dempsey, D. Givord, L. Schultz, and O. Gutfleisch, Scr. Mater. 60, 826 (2009).

${ }^{17}$ W. Szmaja, J. Magn. Magn. Mater. 301, 546 (2006).

${ }^{18}$ W. D. Corner and M. J. Hawton, J. Magn. Magn. Mater. 72, 59 (1988).

${ }^{19}$ W. Szmaja, Czech. J. Phys. 54, 1503 (2004).

${ }^{20}$ J. Y. Wang, L. H. Lewis, D. O. Welch, and P. Canfield, Mater. Charact. 41, 201 (1998).

${ }^{21}$ T. Kohashi, K. Motai, T. Nishiuchi, T. Maki, and S. Hirosawa, J. Magn. Soc. Jpn. 33, 374 (2009).

${ }^{22}$ R. K. Mishra and R. W. Lee, Appl. Phys. Lett. 48, 733 (1986).

${ }^{23}$ M. Uehara, T. Tomida, H. Tomizawa, S. Hirosawa, and Y. Maehara, J. Magn. Magn. Mater. 159, L304 (1996).

${ }^{24}$ Y. Zhu and M. R. McCartney, J. Appl. Phys. 84, 3267 (1998).

${ }^{25}$ Y. Shinba, T. J. Konno, K. Ishikawa, K. Hiraga, and M. Sagawa, J. Appl. Phys. 97, 053504 (2005).

${ }^{26}$ Y.-G. Park and D. Shindo, J. Electron Microsc. 53, 43 (2004).

${ }^{27}$ K. Ono, T. Araki, M. Yano, N. Miyamoto, T. Shoji, A. Kato, A. Manabe, H. Nonaki, Y. Kaneko, and J. Raabe, IEEE Trans. Magn. 47, 2672 (2011).

${ }^{28}$ K. Ono, M. Yano, T. Araki, A. Manabe, N. Miyamoto, T. Shoji, A. Kato, Y. Kaneko, H. Nonaki, and J. Raabe, "22nd International Workshop on Rare-Earth Permanent Magnets and Their Applications," REPMf12 (2012).

${ }^{29}$ C. Herring and C. Kittel, Phys. Rev. 81, 869 (1951). 